\title{
Genomic analysis offers insights into the evolution of the bovine TRA/TRD locus
}

\author{
Timothy K Connelley ${ }^{1 *}$, Kathryn Degnan ${ }^{1}$, Cassandra W Longhi ${ }^{2}$ and W Ivan Morrison ${ }^{1}$
}

\begin{abstract}
Background: The TRA/TRD locus contains the genes for V(D)J somatic rearrangement of TRA and TRD chains expressed by $a \beta$ and $\gamma \delta T$ cells respectively. Previous studies have demonstrated that the bovine TRA/TRD locus contains an exceptionally large number of TRAV/TRDV genes. In this study we combine genomic and transcript analysis to provide insights into the evolutionary development of the bovine TRA/TRD locus and the remarkable TRAV/TRDV gene repertoire.

Results: Annotation of the UMD3.1 assembly identified 371 TRAV/TRDV genes (distributed in 42 subgroups), 3 TRDJ, 6 TRDD, 62 TRAJ and single TRAC and TRDC genes, most of which were located within a $3.5 \mathrm{Mb}$ region of chromosome 10. Most of the TRAV/TRDV subgroups have multiple members and several have undergone dramatic expansion, most notably TRDV1 (60 genes). Wide variation in the proportion of pseudogenes within individual subgroups, suggest that differential 'birth' and 'death' rates have been used to form a functional bovine TRAV/TRDV repertoire which is phylogenetically distinct from that of humans and mice. The expansion of the bovine TRAV/ TRDV gene repertoire has predominantly been achieved through a complex series of homology unit (regions of DNA containing multiple gene) replications. Frequent co-localisation within homology units of genes from subgroups with low and high pseudogene proportions suggest that replication of homology units driven by evolutionary selection for the former may have led to a 'collateral' expansion of the latter. Transcript analysis was used to define the TRAV/ TRDV subgroups available for recombination of TRA and TRD chains and demonstrated preferential usage of different subgroups by the expressed TRA and TRD repertoires, indicating that TRA and TRD selection have had distinct impacts on the evolution of the TRAV/TRDV repertoire.
\end{abstract}

Conclusion: Both TRA and TRD selection have contributed to the evolution of the bovine TRAV/TRDV repertoire. However, our data suggest that due to homology unit duplication TRD selection for TRDV1 subgroup expansion may have substantially contributed to the genomic expansion of several TRAV subgroups. Such data demonstrate how integration of genomic and transcript data can provide a more nuanced appreciation of the evolutionary dynamics that have led to the dramatically expanded bovine TRAV/TRDV repertoire.

Keywords: Bovine, TRA/TRD locus, TR, Duplication, Evolution

\section{Background}

Adaptive immunity in jawed vertebrates is mediated by $B$ cells and T cells, two lymphocyte subsets characterised by the expression of highly diverse repertoires of heterodimeric antigen-specific receptors - immunoglobulins (IG or B cell receptors) and T cell receptors (TR). Immunoglobulins are formed from heavy (IgH) and light

\footnotetext{
* Correspondence: timothy.connelley@ed.ac.uk

${ }^{1}$ The Roslin Institute and Royal (Dick) School of Veterinary Studies, The University of Edinburgh, Easter Bush, Midlothian EH25 9RG, Scotland, UK Full list of author information is available at the end of the article
}

(either Igk or Ig $\lambda$ ) chains and TR from either $\alpha$ and $\beta$ chains ( $\alpha \beta$ T cells) or $\gamma$ and $\delta$ TR chains ( $\gamma \delta$ T cells).

The variable domains of individual IG and TR chains are formed in lymphocyte precursors by somatic recombination of single discontiguous variable $(\mathrm{V})$, diversity (D - in IgH, TRB and TRD chains only) and joining (J) genes selected from multiple genomic copies of these genes. This recombination is directed by the products of the RAG1/2 genes which introduce double-strand breaks at recombination signal (RS) sequences flanking V, D and $J$ genes [1] that are subsequently repaired by nonhomologous joining of the cleaved V(D)J elements. After 
recombination the $\mathrm{V}(\mathrm{D}) \mathrm{J}$ product is spliced to a relatively invariant constant $(C)$ gene. The different permutations of recombined $V(D) J$ genes used to form individual IG and TR chains generate diversity (combinatorial diversity) that is further amplified by the activities of exonuclease and terminal deoxynucleotide transferase which together modify the germline nucleotide sequence at the $\mathrm{V}(\mathrm{D}) \mathrm{J}$ junction (junctional diversity). Together these mechanisms enable the generation of hugely diverse IG and TR repertoires for example, the potential repertoire of unique human $\alpha \beta$ TR has been calculated to be $10^{15}$ [2]. Highly diverse IG and TR repertoires are crucial to facilitating recognition of the vast array of antigens to which hosts may be exposed and are therefore considered critical to an effective adaptive immune system [3].

The constituent genes used to construct TR chains are located in 3 distinct chromosomal loci - TRB for TRB, TRG for TRG and TRA/TRD for both TRA and TRD chains. Sequencing of murine and human TR loci has enabled the full complement of the TR genes in these species to be described (e.g. [4]) and allowed the organisation and regulation of these immunologically significant loci to be analysed (reviewed in [5,6]). Additionally, interspecies and inter-loci comparisons have provided insights into how these loci have evolved (e.g. [7]). Between mammalian species the orthologous TR loci generally demonstrate conservation of overall structure - for example the organisation of genes in the TRB locus is similar in humans [4], mice [7], cattle [8] and dog [9]. Despite this conservation there are marked inter-species differences in both the total number of $\mathrm{V}$ genes and the relative representation of individual $\mathrm{V}$ gene subgroups (genes sharing $\geq 75 \%$ nucleotide identity) located in orthologous loci, indicating that dynamic evolutionary development of $\mathrm{V}$ gene germline repertoires has occurred post-speciation. TR V genes follow a 'birth and death' model of evolution whereby new genes are generated by repeated duplication (and form the substrate for diversification by mutation), of which some are retained whilst others are deleted or become pseudogenes following acquisition of deleterious mutations [10-12]. Dramatic evolutionary modifications of functional $\mathrm{V}$ gene germline repertoires are assumed to reflect selective pressures exerted on the adaptive immune system and in turn result in beneficial changes in the expressed TR repertoires.

The TRA/TRD locus is unique in containing the TR gene elements for 2 TR chains. In humans the TRA/ TRD locus spans $\sim 1 \mathrm{Mb}$ and contains 49 TRAV genes, 5 dual usage TRAV/TRDV genes, 3 TRDV, 3 TRDD, 61 TRAJ, 4 TRDJ and single TRAC and TRDC genes, whilst in mice the TRA/TRD locus occupies $\sim 1.7 \mathrm{Mb}$ and contains 88 TRAV, 10 dual usage TRAV/TRDV genes, 6 TRDV, 2 TRDD, 60 TRAJ, 2 TRDJ and single TRAC and TRDC genes [13]. Comparative genomics show that the human and murine TRA/TRD loci share a similar gene organisation with most of the $\mathrm{V}$ gene segments located at the $5^{\prime}$ end of the locus and the other TRA/TRD genes and a single TRDV gene with an inverted orientation located at the 3' end [7]. Two studies have analysed the genomic sequence of the bovine TRA/TRD locus $[14,15]$. However, these studies were largely restricted to cataloguing the repertoires of either TRD [14] or TRAV/ TRDV [15] genes, and an annotation of all of the TR genes within the locus has yet to be completed. Furthermore, although a main finding in both studies was the dramatic expansion of the $\mathrm{V}$ gene repertoires (a total of $>400$ TRAV/TRDV genes were identified), analysis of the duplication events that generated this hugely expanded germline repertoire, which could inform our understanding of how the bovine TRAV/TRDV repertoire has evolved, have not been conducted.

In this study, we use the most recent assembly of the bovine genome (UMD3.1 [16]) to re-examine the genomic sequence of the TRA/TRD locus. We sought to complete a comprehensive annotation of the TRA/TRD gene repertoire and use this as a basis to fulfil our primary aim of examining the evolutionary development of the massive expansion of bovine TRAV/TRDV genes. By combining analysis of the genome assembly and TRA/TRD transcripts we present data indicating that i) the majority of the TRAV/TRDV gene expansion is attributable to a complex series of duplications of multiple homology units (i.e. regions of DNA incorporating multiple genes), ii) expansion of some TRAV subgroups may have occurred 'collaterally', as a consequence of selection for expanded functional repertoires of other subgroups (most notably TRDV1), and iii) the expressed TRA and TRD repertoires preferentially use different TRAV/TRDV subgroups, suggesting TRA and TRD selection have made distinct contributions to the evolutionary development of the genomic TRAV/TRDV repertoire.

\section{Results}

\section{Annotation of the TRA/D gene repertoire}

A prerequisite of our study was accurate, up-to-date information on the bovine genomic TRA/TRD gene repertoire. Annotation of the UMD3.1 bovine genome assembly identified a total of 371 TRAV/TRDV, 3 TRDJ, 6 TRDD, 62 TRAJ, 1 TRAC and 1 TRDC genes. All of these TR genes, with the exception of 12 TRAV/TRDV genes, were located within a $3.5 \mathrm{Mb}$ region of chromosome 10 (Additional file 1).

Phylogenetic analysis indicated the existence of 42 distinct bovine TRAV/TRDV gene subgroups (Additional file 2). Of these, 35 were orthologous to human TRAV subgroups and 3 to human TRDV subgroups and therefore were provisionally assigned the corresponding numbers (see Methods). Of the remaining 4 subgroups, one 
contained a single gene with $73.3 \%$ nucleotide identity with murine TRDV4 and another contained 7 genes showing 72.7-75.8\% nucleotide identity with murine TRAV4 - these were designated as bTRDVY and bTRAVY respectively. The other 2 subgroups lacked directly orthologous human or murine subgroups. Genes from 1 of these subgroups had been previously described as bTRDV3 genes [14,17] - we have re-assigned this subgroup as bTRDVb3 to distinguish it from the bovine TRDV3 subgroup. Subsequent cDNA analyses (see below) demonstrated that members of the final subgroup recombined with TRAJ genes - we consequently designated this subgroup as bTRAVX. All but 8 of the TRAV/TRDV subgroups (TRAV1, 27, 35, 36, 41, and TRDV2, 3 and Y) are multi-membered and several subgroups (TRAV22, 23, 25, 26, $\mathrm{X}$ and TRDV1) have undergone dramatic expansion, containing more than 20 members (Table 1).

Although our findings are broadly consistent with the findings from previous annotations $[14,15]$ there are several features that make this annotation distinct: i) the number of TRAV/TRDV genes identified is lower than in previous annotations (Additional file 3), ii) several novel subgroups have been identified, iii) the full length (L exon, $\mathrm{V}$ exon and RS) of all TRAV/TRDV genes has been annotated (Additional file 4 and Additional file 5), and iv) all of the TRA/TRD genes have been included. Notably only about half of the TRAV/TRDV genes identified here had $100 \%$ sequence identity to genes identified in the Btau_4.0 assembly (Additional file 3).

\section{The structure of the 3' end of the bovine TRA/TRD locus shows syntenic conservation with that of humans and mice}

The UMD3.1 assembly of the 3' end of the bovine TRA/ TRD locus appears to be essentially complete. All TRAC, TRAJ, TRDC, TRDJ, TRDD genes and the TRDV3 gene (in inverted orientation) are located on 2 large contigs (DAAA02028052.1 - $140 \mathrm{~Kb}$ and DAAAA02028053.1 $114 \mathrm{~Kb})$ separated by a gap of approximately $1 \mathrm{~Kb}$. The organisation of the genes demonstrates a marked syntenic conservation between mouse, human and bovine (Figure 1). Synteny is maintained within the TRAJ repertoire with individual bovine TRAJ genes sharing a conserved order with their murine and human orthologues (Additional file 6). Additionally there is conservation of important regulatory elements at the 3 ' end of the TRA/ TRD locus, with bovine sequences showing identity with the human/murine TRA enhancer $(E \alpha)$, TRD enhancer (ES), CSB and TEA located in syntenic positions (Figure 1 and Additional file 1 and Additional file 7). In addition to gene content and organisation, the size of the 3' end of TRA/TRD locus is similar across the 3 species (approximately $100 \mathrm{~Kb}$ from TRDJ to TRAC), with the exception of the TRDD region which in bovine is $\sim 80 \mathrm{~Kb}-$ approximately $8 \times$ the size of the equivalent regions in the mouse and human TRA/TRD loci.

\section{Expansion of the bovine TRAV/TRDV repertoire has predominantly occurred via duplication of homology units}

The UMD3.1 assembly of the $5^{\prime}$ end of the locus is highly fragmented with the $\sim 3.3 \mathrm{Mb}$ of sequence distributed across $>250$ separate contigs. Although this fragmentation precludes a description of the overall genomic structure of the $5^{\prime}$ end of the locus, regions of substantial contiguous sequence (up to $198 \mathrm{~Kb}$ ) enable examination of the organisation of sections of the TRAV/TRDV region. Within these sections small areas demonstrating syntenic conservation with human TRAV/TRDV genes were identified (indicated by juxtaposition of genes from consecutively numbered TRAV/TRDV subgroups - Additional file 1). However, a more prominent feature of the TRAV/TRDV gene organisation within these contigs was recurrent patterns of intercalation of genes from the multimembered subgroups. For example the ordered sequence of TRAV12-11-10-9 genes recurs 6 times in the UMD3.1 assembly (Additional file 1), suggesting that replication of DNA segments containing multiple TRAV/TRDV genes has led to the formation of 'homology units'. Detailed nucleotide identity analysis of these regions indicated the presence of 10 putative homology units incorporating between 2 and 17 TRAV/TRDV genes and extending in size from $\sim 15 \mathrm{~Kb}$ to $>150 \mathrm{~Kb}$ (Table 2 and Additional file 8).

Within the context of homology unit replication the expansion of some multi-membered subgroups appears evolutionarily simple. For example, amplification of the TRAV2 and 3 subgroups is due to 6 tandem replications of homology unit 2, resulting in a $\sim 140 \mathrm{~Kb}$ region with alternating TRAV2 and TRAV3 genes at the $5^{\prime}$ end of the locus. In contrast, expansion of other subgroups, especially those that have undergone prolific expansion, appears to be evolutionarily complex. TRDV1 subgroup members are found on 4 different homology units (homology units 7, 8, 9 and 10), intercalated with different permutations of genes including members of the TRAV22, 23, 25 and 26 subgroups. The presence of multiple copies of genes from some subgroups (e.g. up to 3 copies of TRDV1 in homology unit 9) amplifies the expansive effect of homology unit replication on the size of these subgroups. Notably TRAVX expansion is also largely a product of members being incorporated into multiple homology units (units 5 and 6) and the presence of multiple members of this subgroup being present in the homology units. Most of the homology units have undergone multiple replicative events (Table 2) and in some cases variable proportions of the unit appear to have been duplicated (e.g. homology units 5.6 and 5.7 appear to represent a variant in which the TRAV9-14-13-Y-9 component 
Table 1 The human and bovine (UMD3.1) TRAV/TRDV repertoires

\begin{tabular}{|c|c|c|c|c|c|c|c|c|c|}
\hline \multirow[t]{2}{*}{ Subgroup } & \multicolumn{4}{|c|}{ Human } & \multicolumn{5}{|c|}{ Bovine } \\
\hline & Total & $\begin{array}{c}\text { Functional } \\
\text { (Number of ge }\end{array}$ & $\begin{array}{l}\text { Pseudo. } \\
\text { s) }\end{array}$ & $\begin{array}{l}\text { Percentage } \\
\text { functional }\end{array}$ & Total & $\begin{array}{l}\text { Functional } \\
\text { (Numk }\end{array}$ & $\begin{array}{l}\text { Pseudo. } \\
\text { of genes) }\end{array}$ & Incomplete & $\begin{array}{l}\text { Percentage } \\
\text { functional }\end{array}$ \\
\hline$A V 1$ & 2 & 2 & & 100 & 1 & 1 & & & 100 \\
\hline AV2 & 1 & 1 & & 100 & 7 & 5 & 2 & & 71.4 \\
\hline AV3 & 1 & $1+$ & $1+$ & $100 / 0$ & 7 & 7 & & & 100 \\
\hline AV4 & 1 & 1 & & 100 & 3 & 2 & 1 & & 66.7 \\
\hline AV5 & 1 & 1 & & 100 & 2 & 2 & & & 100 \\
\hline AV6 & 1 & 1 & & 100 & 4 & & 3 & 1 & 0 \\
\hline AV7 & 1 & 1 & & 100 & & & & & \\
\hline AV8 & 7 & 5 & 2 & 71.4 & 14 & 6 & 8 & & 42.9 \\
\hline AV9 & 2 & 2 & & 100 & 11 & 1 & 9 & 1 & 9.1 \\
\hline AV10 & 1 & 1 & & 100 & 6 & 3 & 3 & & 50 \\
\hline AV11 & 1 & & 1 & 0 & 6 & & 6 & & 0 \\
\hline AV12 & 3 & 3 & & 100 & 6 & 5 & & 1 & 83.3 \\
\hline AV13 & 2 & 2 & & 100 & 11 & 8 & 2 & 1 & 72.8 \\
\hline AV14 & 1 & 1 & & 100 & 9 & 4 & 4 & 1 & 44.4 \\
\hline AV15 & 1 & & 1 & 0 & & & & & \\
\hline AV16 & 1 & 1 & & 100 & 2 & 2 & & & 100 \\
\hline AV17 & 1 & 1 & & 100 & 3 & 1 & 2 & & 33.3 \\
\hline AV18 & 1 & 1 & & 100 & 8 & 3 & 5 & & 37.5 \\
\hline AV19 & 1 & 1 & & 100 & 7 & 5 & 2 & & 71.4 \\
\hline AV20 & 1 & 1 & & 100 & 5 & 5 & & & 100 \\
\hline AV21 & 1 & 1 & & 100 & 4 & 3 & 1 & & 75 \\
\hline AV22 & 1 & 1 & & 100 & 29 & 14 & 14 & 1 & 48.3 \\
\hline AV23 & 1 & 1 & & 100 & 24 & 4 & 20 & & 16.7 \\
\hline AV24 & 1 & 1 & & 100 & 10 & 1 & 8 & 1 & 10 \\
\hline AV25 & 1 & 1 & & 100 & 22 & 11 & 10 & 1 & 50 \\
\hline AV26 & 2 & 2 & & 100 & 39 & 22 & 13 & 4 & 56.4 \\
\hline AV27 & 1 & 1 & & 100 & 1 & 1 & & & 100 \\
\hline AV28 & 1 & & 1 & 0 & 4 & 3 & 1 & & 75 \\
\hline AV29 & 1 & $1+$ & $1+$ & $100 / 0$ & 3 & 2 & 1 & & 66.7 \\
\hline AV30 & 1 & 1 & & 100 & & & & & \\
\hline AV31 & 1 & & 1 & 0 & & & & & \\
\hline AV32 & 1 & & 1 & 0 & & & & & \\
\hline AV33 & 1 & & 1 & 0 & 6 & 2 & 4 & & 33.3 \\
\hline AV34 & 1 & 1 & & 100 & 3 & & 3 & & 0 \\
\hline AV35 & 1 & 1 & & 100 & 1 & 1 & & & 100 \\
\hline AV36 & 1 & 1 & & 100 & 1 & 1 & & & 100 \\
\hline AV37 & 1 & & 1 & 0 & 2 & & 2 & & 0 \\
\hline AV38 & 2 & 2 & & 100 & 6 & 5 & & 1 & 83.3 \\
\hline AV39 & 1 & 1 & & 100 & 2 & 1 & 1 & & 50 \\
\hline AV40 & 1 & 1 & & 100 & & & & & \\
\hline AV41 & 1 & 1 & & 100 & 1 & 1 & & & 100 \\
\hline AVX & & & & & 29 & 23 & 5 & 1 & 79.3 \\
\hline
\end{tabular}


Table 1 The human and bovine (UMD3.1) TRAV/TRDV repertoires (Continued)

\begin{tabular}{|c|c|c|c|c|c|c|c|c|c|}
\hline AVY & & & & & 7 & 7 & & & 100 \\
\hline DV1 & 1 & 1 & & 100 & 60 & 48 & 8 & 4 & 80.0 \\
\hline DV2 & 1 & 1 & & 100 & 1 & 1 & & & 100 \\
\hline DV3 & 1 & 1 & & 100 & 1 & 1 & & & 100 \\
\hline DVb3 & & & & & 2 & 2 & & & 100 \\
\hline DVY & & & & & 1 & 1 & & & 100 \\
\hline Total & 57 & $46(+2)$ & $9(+2)$ & 80.7 & 371 & 215 & 138 & 18 & 58.0 \\
\hline
\end{tabular}

The numbers of total, functional and pseudogenes in each TRAV/TRDV subgroup in the germline human and bovine (UMD3.1) repertoires are shown. The percentage of genes in each subgroup that are functional has been calculated (for human genes that have alternative functional and non-functional alleles denoted by $\dagger$, two percentage values are shown). For the bovine TRAV/TRDV subgroups, the number of genes for which only incomplete genomic sequence was available is also shown. Details of the human TRAV/TRDV repertoire were taken from the IMGT database (http://www.imgt.org).

of the homology unit has been excluded - Additional file 9) whilst others display evidence of post-replication deletion/insertion events modifying the gene content of some replicons. For example, the 2 copies of homology unit 1 show disparity in the number of TRAV28 genes present (Additional file 9).

The location of 288 out of the 371 (77.6\%) TRAV/TRDV genes identified in the UMD3.1 assembly within homology units attests to replication of homology units being the principal mechanism employed in achieving the massively expanded bovine TRAV/TRDV repertoire. The multiplicity of identified homology units, the variation in replicon number and the modifications of homology unit content during and after replication suggest the evolutionary development of the bovine TRAV/TRDV repertoire has been highly complex.

Extensive duplication of the TRDV1 subgroup has skewed the phylogenetic representation of the functional TRAV/ TRDV gene repertoire of cattle

The functional competency of $\mathrm{V}, \mathrm{D}$ and $\mathrm{J}$ genes is dependent on the maintenance of a correct open reading frame (ORF), the presence of codons encoding certain critical amino acid residues and the presence of a RS. In silico sequence analysis based on these parameters indicates that all TRDD and TRDJ genes and 52 out of 62 (83.9\%) of TRAJ genes are functional (Additional file 10) and so the repertoire size of these genes is fairly comparable to that seen in mice and humans (Table 3 ).

Of the 371 TRAV/TRDV genes, 215 (58.0\%) were predicted to encode functional products and 138 (37.2\%) pseudogenes (Additional file 11), whilst the functional competency of the remaining 18 (4.8\%) genes could not be assessed as the sequences were incomplete (Table 1). The predicted bovine functional TRAV/TRDV repertoire incorporates genes from 38 subgroups and is approximately 2.5 and 4 times larger than that of mice and humans respectively. Between multi-membered subgroups there is huge disparity in the proportion of genes that are functional - some having a high proportion of pseudogenes (e.g. TRAV9 and TRAV11) whilst in others (e.g. TRAV3 and TRAVY) every gene is predicted to be functional. This extends to the massively expanded subgroups, with TRAVX and TRDV1 subgroups retaining a

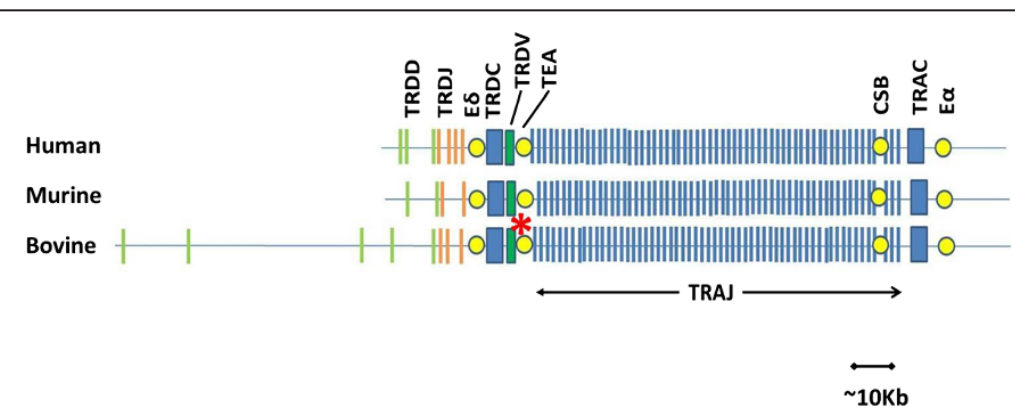

Figure 1 Conserved synteny of the $3^{\prime}$ end of the bovine, human and murine TRA/TRD loci. A schematic representation shows the conserved inter-species organisation of TR genes and regulatory elements at the $3^{\prime}$ end of the TRA/TRD locus. The approximate positions of TRDD (green lines), TRDJ (orange lines), TRAJ (blue lines), TRDC and TRAC (blue blocks), the single inverted TRDV gene (TRDV3 in bovine and human, TRDV5 in mouse - green block) and regulatory elements (yellow circles) are shown. The regulatory elements represented are - TRA enhancer (Ea), TRD enhancer (ES), T early alpha (TEA) and conserved sequence block (CSB). The length of the region is also conserved across the species, with the exception of the area occupied by TRDD genes, which is much greater in the bovine TRA/TRD locus ( $80 \mathrm{~Kb})$ than in the mouse or human ( 10 Kb). The asterisk indicates the approximate location of the $1 \mathrm{~Kb}$ gap between contigs DAAA02028052.1 and DAAAA02028053.1. 
Table 2 Putative bovine TRAV/TRDV gene homology units identified in the UMD3.1 assembly

\begin{tabular}{|c|c|c|c|c|c|c|}
\hline \multirow{2}{*}{$\begin{array}{l}\text { Homology } \\
\text { unit }\end{array}$} & \multirow[t]{2}{*}{ TRAV/TRDV gene motif } & \multirow{2}{*}{$\begin{array}{l}\text { No. of TRAV/ } \\
\text { TRDV genes }\end{array}$} & \multirow[t]{2}{*}{ Size (Kb) } & \multirow{2}{*}{$\begin{array}{l}\text { Number of } \\
\text { replicons }\end{array}$} & \multicolumn{2}{|c|}{ Variable gene content } \\
\hline & & & & & $\begin{array}{c}\text { Proportion of homology } \\
\text { unit amplified }\end{array}$ & $\begin{array}{c}\text { Post-replication } \\
\text { insertion/deletion }\end{array}$ \\
\hline 1 & $33-29-28-33-34-26-33$ & 7 & 39 & 2 & & Yes \\
\hline 2 & $2-3$ & 2 & 15 & 7 & & \\
\hline 3 & $38-37$ & 2 & 15 & 2 & & \\
\hline 4 & $14-13-9$ & 3 & 15 & 5 & & \\
\hline 5 & $22-8-21-20-19-X-X-X-18-12-11-10-9-14-13-Y-9$ & $17(15)$ & 157 & 6 & Yes & \\
\hline 6 & $25-19-X-X-X-X-18-17-16$ & $9(8)$ & 80 & 3 & & \\
\hline 7 & DV1-22-26-25-DV1-23-22-8-21-20 & 10 & 71 & 5 & & \\
\hline 8 & DV1-23-22-26-DV1-25-23-22-26-DV1-25 & 11 & 63 & 8 & Yes & Yes \\
\hline 9 & DV1-22-8-DV1-26-25-24-DV1-26-DV1-26 & $12(8)$ & 86 & 7 & Yes & Yes \\
\hline 10 & DV1-23-22-24-DV1-23 & 6 & 43 & 2 & & \\
\hline
\end{tabular}

For each homology unit the TRAV/TRDV gene motif, the number of genes, the estimated minimal size and the number of replicons identified are shown. Due to the fragmentary nature of the UMD3.1 assembly many of the homology units presented here and many of the copies of the homology units are likely to be incomplete. Therefore the TRAV/TRDV gene motif, number of TRAV/TRDV genes and size of homology units should be considered a minimum. Similarly, we have rationalised the number of replicons for each homology unit to the minimum that could account for all of the partial replications observed in the UMD3.1 assembly and so this should also be considered a minimum. For homology units 5,6 and 9 no single copy covers the entirety of the homology unit and we have estimated its size by combining data from multiple copies; the number of genes in the most extensive copy of these homology units is indicated in parentheses in the 'No. of TRAV/TRDV genes' column. In addition we have summarised where there is evidence in the UMD3.1 assembly of the TRAV/TRDV gene content of copies of a homology unit differing due to either varying proportions of the homology unit being duplicated in different replicative iterations or post-replication insertion/deletion (see Additional file 8).

high proportion of functional genes $(\sim 80 \%)$ and TRAV23 having a low proportion $(\sim 15 \%)$. Notably members of subgroups with high proportions of pseudogenes are generally co-localised within homology units with genes from subgroups with low pseudogene proportions: for example many TRAV23, 24 and 8 pseudogenes are found with TRDV1 genes on homology units 7-10 (Additional file 9). Such juxtaposition suggests that homology unit replication due to selection for expansion of some subgroups (e.g. TRDV1) may have co-incidentally led to the expansion of other TRAV/TRDV subgroups (e.g. TRAV23, 24 and 8) for which there has been no evolutionary selection to generate/maintain an expanded functional genomic repertoire.

Neighbour-joining analysis resolved functional murine, human and bovine TRAV/TRDV genes into 4 monophylogenic groups (Figure 2). In mice and humans there is marked inter-species similarity in the distribution between

Table 3 Repertoire of functional TRA/TRD gene segments in the bovine (UMD3.1), murine and human genomes

\begin{tabular}{cccc}
\hline & Bovine (UMD3.1) & Human & Murine \\
\hline TRAV/TRDV & $215(38)$ & $48^{*}(37)$ & $87^{*}(23)$ \\
TRAJ & 52 & 50 & 38 \\
TRDD & 5 & 3 & 2 \\
TRDJ & 3 & 4 & 2 \\
\hline
\end{tabular}

*For human and murine TRAV/TRDV repertoires we represent the maximum number of potential functional genes (i.e. including TRAV/TRDV gene segments for which both functional and non-functional alleles exist as functional). The numbers in parentheses show the number of TRAV/TRDV subgroups that include functional members. these monophylogenic groups, with Groups 1, 2, 3 and 4 containing approximately $50 \%, 35 \%, 1 \%$ and $10 \%$ of the functional TRAV/TRDV genes respectively in both species (Figure 2B). In contrast, the distribution of functional genes in bovine is different with a relative contraction of Group 1 (34.4\%) and expansion of Group 4 (30.7\%); the latter due to the high number of TRDV1 genes. In contrast to mice and humans, in which the relative proportions of the 4 Groups within the total gene repertoires (Figure 2C) are similar to that observed in the functional repertoires, in bovine the representation of Group 1 is higher (42.3\%) and that of Group 4 is lower $(24.3 \%)$ in the total than in the functional repertoires. Thus, it is apparent that massive expansion of particular TRAV/TRDV subgroups (most prominently TRDV1) combined with the preferential retention of functionality in some of these subgroups act synergistically to cause a profound phylogenetic shift in the repertoire of functional TRAV/TRDV genes available for rearrangement of TRA/TRD chains.

\section{Analysis of expressed TRA and TRD repertoires suggests preferential usage of particular TRAV/TRDV subgroups} Although the expressed bovine TRD chain repertoire has been examined extensively in a number of studies [17-21], the published data on the expressed bovine TRA chain repertoire is limited [22,23]. A search of the NCBI EST archive revealed a restricted number of entries for rearranged TRA chains (80 entries including representatives from 24 different TRAV/TRDV subgroups Table 4). To facilitate a direct examination of the TRAV/ 


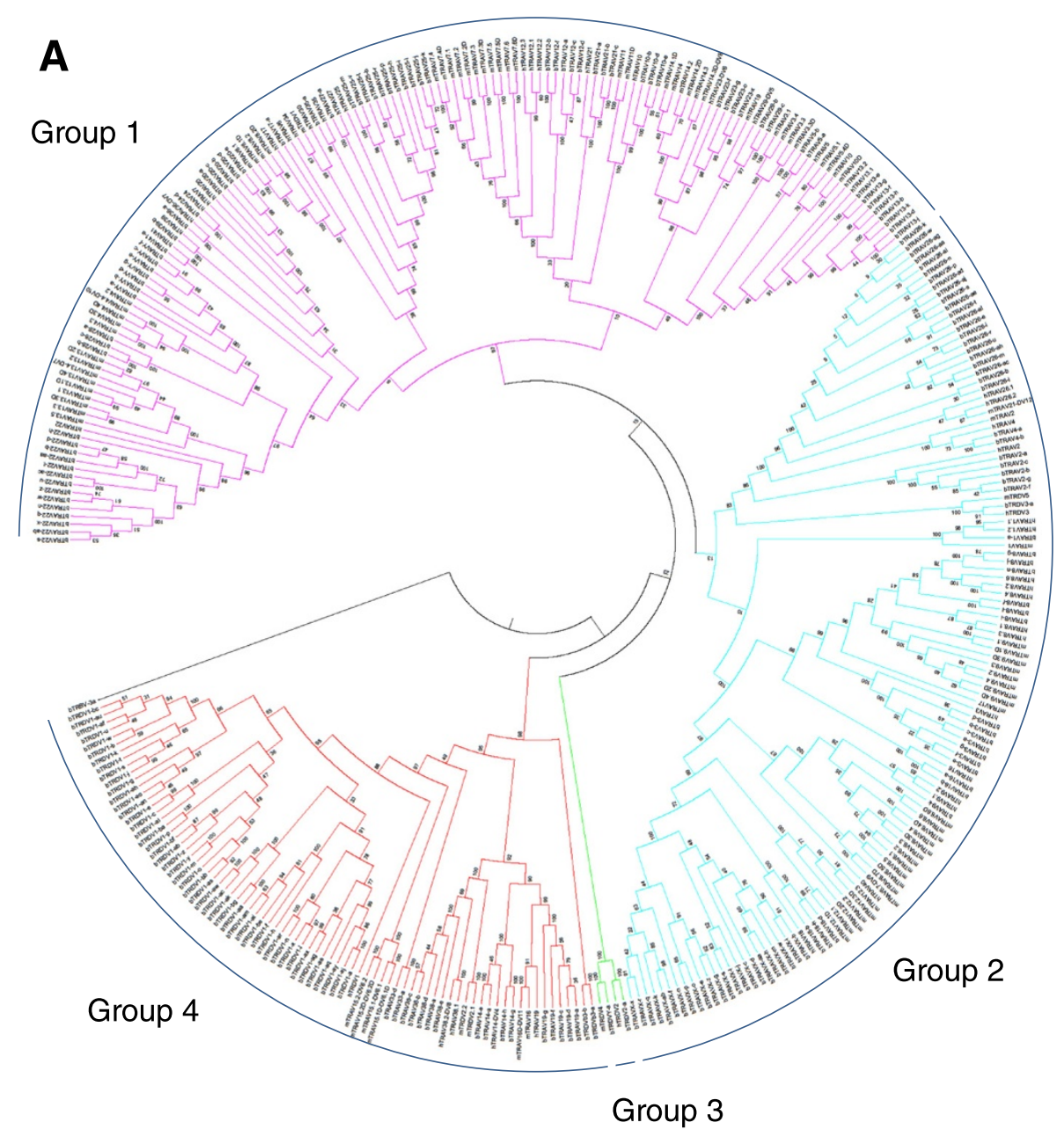

B

\begin{tabular}{|r|r|r|r|}
\hline & Mouse & Human & Bovine \\
\hline Group1 & 54.0 & 50.0 & 34.4 \\
\hline Group2 & 35.6 & 37.5 & 34.0 \\
\hline Group3 & 1.1 & 2.1 & 0.9 \\
\hline Group4 & 9.2 & 10.4 & 30.7 \\
\hline
\end{tabular}

\section{C}

\begin{tabular}{|r|r|r|r|}
\hline & Mouse & Human & Bovine \\
\hline Group1 & 57.7 & 50.9 & 42.3 \\
\hline Group2 & 31.7 & 35.1 & 32.9 \\
\hline Group3 & 1.0 & 1.8 & 0.5 \\
\hline Group4 & 9.6 & 12.3 & 24.3 \\
\hline
\end{tabular}

Figure 2 Phylogenetic analysis of the repertoires of functional TRAV/TRDV genes in mice, humans and cattle. (A) - Neighbour-joining phylogenetic tree of all functional murine, human and bovine (from the UMD3.1 assembly) TRAV/TRDV genes. Analysis of the nucleotide sequence of the V-REGION (IMGT nomenclature) following pairwise deletion to remove gaps in the alignment. The final dataset included 340 positions. The sequence of bTRBV3a was used to root the tree. Based on 1000 boot strap replicates support for Groups 1 (purple), 3 (green) and 4 (red) was high with $P_{B}>97 \%$. Support for Group 2 (blue) was low $\left(P_{B}=13 \%\right.$ ) but examination of the data using UPMGA and minimum evolution models generated the same phylogenic groups, suggesting it was reliable (data not shown). $h=$ human, $b=$ bovine and $m=$ murine. Percentage of the functional (B) and total (C) TRAV/TRDV genes in humans, mice and cattle in the 4 phylogenetic Groups defined from neighbour-joining analysis.

TRDV subgroups contributing to the expressed TRA and TRD chain repertoires, we designed a panel of TRAV/TRDV subgroup-specific $5^{\prime}$ primers that could be used in conjunction with TRAC- and TRDCspecific 3' primers to amplify rearranged TRA and TRD chains from cDNA. Sequencing of PCR amplicons generated from $\gamma \delta$ T cells and $\alpha \beta$ T cells demonstrated that; i) all of the TRAV subgroups predicted to include functional members (with the exception of TRAV39) and also TRDV1, TRDV3 and TRDVb3 could rearrange to form functional TRA chains and ii) genes from all of the TRDV subgroups and TRAV33 can functionally rearrange with TRDC. Thus, the expressed TRA and TRD chain repertoires can utilise genes from a minimum of 35 and 6 TRAV/TRDV subgroups respectively. 
Table 4 Summary of EST and PCR data on bovine TRAV/TRDV subgroup expression in re-arranged TRA and TRD chains

\begin{tabular}{|c|c|c|c|c|c|c|c|c|}
\hline \multirow{3}{*}{$\begin{array}{c}\text { TRAV/ } \\
\text { TRDV } \\
\text { subgroup }\end{array}$} & \multirow{2}{*}{\multicolumn{2}{|c|}{$\begin{array}{l}\text { EST evidence of use in } \\
\text { TRA/TRD rearrangement }\end{array}$}} & \multicolumn{6}{|c|}{ PCR data (iii) } \\
\hline & & & \multirow{2}{*}{$\begin{array}{l}\text { No. of unique } \\
\text { TRA/TRD chain } \\
\text { sequences (iv) }\end{array}$} & \multirow{2}{*}{$\begin{array}{c}\text { Total number of } \\
\text { unique TRAV/TRDV } \\
\text { genes }(v)\end{array}$} & \multicolumn{2}{|c|}{ Identical genome matches } & \multirow{2}{*}{$\begin{array}{l}\text { No. of matches } \\
>97 \% \text { (viii) }\end{array}$} & \multirow{2}{*}{$\begin{array}{l}\text { No. of matches } \\
<97 \% \text { (ix) }\end{array}$} \\
\hline & TRA (i) & TRD (ii) & & & Number (vi) & $\begin{array}{l}\text { Matching TRAV/ } \\
\text { TRDV gene (vii) }\end{array}$ & & \\
\hline AV1 & $\bullet$ & & 5 & 3 & 1 & A & 2 & \\
\hline $\mathrm{AV} 2$ & & & 4 & 3 & 1 & G & 2 & \\
\hline $\mathrm{AV} 3$ & $\bullet$ & & 13 & 8 & 5 & $a, b, c, e, f$ & 3 & \\
\hline AV4 & & & 2 & 2 & - & & 2 & \\
\hline AV5 & $\bullet$ & & 1 & 1 & - & & & 1 \\
\hline AV8 & $\bullet$ & & 9 & 7 & 3 & $\mathrm{I}, \mathrm{l}, \mathrm{n}$ & 3 & 1 \\
\hline AV9 & $\bullet$ & & 2 & 2 & 1 & K & 1 & \\
\hline AV10 & $\bullet$ & & 5 & 3 & 1 & B & 2 & \\
\hline AV12 & $\bullet$ & & 10 & 7 & 1 & D & 5 & 1 \\
\hline AV13 & $\bullet$ & & 8 & 7 & 3 & $f, j, k$ & 3 & 1 \\
\hline AV14 & & & 2 & 2 & 1 & $E$ & 1 & \\
\hline AV16 & $\bullet$ & & 4 & 4 & 2 & $a, b$ & 2 & \\
\hline AV17 & $\bullet$ & & 8 & 4 & 2 & $a, c$ & 2 & \\
\hline AV18 & & & 2 & 2 & - & & 2 & \\
\hline AV19 & & & 4 & 2 & 1 & C & 1 & \\
\hline AV20 & & & 3 & 1 & 1 & $E$ & & \\
\hline AV21 & $\bullet$ & & 4 & 2 & 1 & C & 1 & \\
\hline AV22 & $\bullet$ & & 11 & 9 & 2 & aa, w & 6 & 1 \\
\hline AV23 & $\bullet$ & & 3 & 2 & 1 & $x$ & 1 & \\
\hline AV24 & & & 1 & 1 & - & & 1 & \\
\hline AV25 & • & & 5 & 4 & 1 & v & 2 & 1 \\
\hline AV26 & $\bullet$ & & 5 & 5 & 3 & $a j, s, w$ & 2 & \\
\hline AV27 & $\bullet$ & & 5 & 3 & - & & 1 & 2 \\
\hline AV28 & $\bullet$ & & 3 & 2 & 2 & $b, c$ & & \\
\hline AV29 & • & & 8 & 4 & 2 & $a, b$ & 2 & \\
\hline AV33 & & • & $7^{* /} \dagger$ & 6 & - & & 1 & 5 \\
\hline AV34 & & & - & & - & & - & \\
\hline AV35 & $\bullet$ & & 3 & 2 & - & & 2 & \\
\hline AV36 & & & 2 & 1 & 1 & a & & \\
\hline AV38 & & & 10 & 6 & - & & 5 & 1 \\
\hline AV39 & & & - & & & & & \\
\hline AV41 & $\bullet$ & & 1 & 1 & - & & 1 & \\
\hline$A V X$ & $\bullet$ & & 35 & 23 & 11 & $\begin{array}{c}a a, a b, b, f, g, l, p, \\
q, u, w, x\end{array}$ & 12 & \\
\hline AVY & & & 10 & 7 & 3 & $d, e, g$ & 4 & \\
\hline DV1 & $\bullet$ & $\bullet$ & $111^{* /} \dagger$ & 45 & 13 & $\begin{array}{c}\text { af, ah, ai, as, au, b, } \\
\text { bb, e, g, aj, bg, j, } \\
\text { w }\end{array}$ & 25 & 7 \\
\hline DV2 & & & $2+$ & 1 & 1 & a & & \\
\hline DV3 & $\bullet$ & $\bullet$ & $2+$ & 1 & 1 & a & & \\
\hline
\end{tabular}


Table 4 Summary of EST and PCR data on bovine TRAV/TRDV subgroup expression in re-arranged TRA and TRD chains (Continued)

\begin{tabular}{|c|c|c|c|c|c|c|c|}
\hline DVb3 & $\bullet$ & $2+$ & 6 & 1 & $b$ & 5 & \\
\hline DVY & & $29+$ & 2 & 1 & $a$ & 1 & \\
\hline Total & & 341 & 191 & 67 & & 103 & 21 \\
\hline
\end{tabular}

BLAST analysis of the NCBI Bos taurus EST archive (March 2013) using the bovine TRAC and TRDC sequences identified 24 subgroups participating in TRA chain rearrangement (column i) and 4 participating in TRD rearrangement (column ii). Using a combination of bovine TRAV/TRDV subgroup-specific and unbiased $\mathrm{SMART} \mathrm{TRAC}^{+}$and TRDC ${ }^{+}$PCR (column iii) 341 unique full or partial TRA/TRD chain transcripts were sequenced (column iv), providing a more comprehensive description of the breadth of TRAV/TRDV subgroup usage in TRA and TRD transcripts. Amongst these sequences 191 unique TRAV/TRDV gene sequences were identified (column v), of which only 67 (35.1\%) matched any of the genomic sequences identified in the UMD3.1 assembly (column vi and vii). Twenty-one of the TRAV/TRDV gene sequences (11\%) were sufficiently dissimilar to genomic TRAV/TRDV genes ( $<97 \%$ nucleotide identity) to be considered as products of TRAV/ TRDV genes absent from the UMD3.1 assembly (column ix), whilst the other 103 sequences ( $53.9 \%$ ) showed $>97 \%$ identity with genomic sequences and may either represent products of novel TRAV/TRDV genes or allelic variants of the genes in the UMD3.1 assembly (column viii). Despite several attempts no amplification could be achieved with TRAV39 subgroup-specific primers and there was no EST data supporting use of genes from this subgroup in rearranged TRA or TRD chains; consequently this is the only subgroup which was predicted to contain functional TRAV/TRDV genes for which there is no evidence of expression in the TRA/TRD repertoires.

To determine at what frequencies the different TRAV/ TRDV subgroups were utilised in the expressed TRA and TRD repertoires, we used a SMART RACE-PCR system that enables unbiased amplification of TR chain sequences [24] to analyse TRA and TRD transcripts from $\alpha \beta$ and $\gamma \delta$ T cells of 2 MHC-disparate animals. TRA chain analysis showed utilisation of a diverse range of TRAV/TRDV subgroups (19 and 23 subgroups) in both animals (Figure 3A). Although most subgroups were represented at low frequencies $(<5 \%)$, in each animal there was a limited number of subgroups (4 or 5 ) that constituted a higher proportion of the repertoire. Although these highly represented subgroups generally differed between the 2 individuals, both animals showed a particularly high representation of TRAV3 (11.3\% and $8.2 \%$ ) and TRAVX (23\% and 22.6\%). Comparison with the functional genomic TRAV/TRDV gene repertoire revealed that these two TRAV/TRDV subgroups, as well as TRAV17, 27 and 29, were relatively over-represented in the expressed TRA chain repertoire in both animals, whilst TRAV26 and TRDV1 were under-represented (Figure 3A). With reference to the phylogenetic groups discussed above, Groups 1 and 2 each accounted for approximately $\sim 45 \%$ of the expressed TRA chain sequences whilst the remaining $\sim 10 \%$ used genes from Group 4 . Thus, in comparison to the genomic repertoire there is a preference for use of Group 1 and 2 genes and bias against use of Group 4 genes.

As anticipated from the TRAV/TRDV subgroup-specific PCR, the TRD chain repertoire in both animals showed a limited use of TRAV/TRDV subgroups. The TRD chain sequences obtained from both animals were dominated by TRDV1 and TRDVb3, which accounted for $90 \%$ and $10 \%$ of the sequences respectively in one animal and $60 \%$ and $32 \%$ in the other, with only single additional TRDVY and TRAV33 sequences found in the second animal (Figure 3B). The dominance of TRDV1 observed was consistent with results from previous analysis [21]. With the exception of TRDVY, all of the subgroups detected in the sequenced TRD repertoire belong to phylogenetic Group 4 and consequently the TRD repertoire analysed here is almost completely restricted ( $>99 \%)$ to this Group.

During this study, 198 and 143 unique functional TRA and TRD chains, utilizing a total of 191 distinct TRAV/ TRDV genes, were sequenced. Only $67(35.1 \%)$ of the expressed TRAV/TRDV gene sequences were identical to a gene identified from the genome. All of the remainder belonged to already identified subgroups; 21 (11.0\%) of them showed $<97 \%$ identity to one or more genomic sequences (the level of identity conventionally used in mouse and humans to distinguish products of distinct TR $\mathrm{V}$ genes $[25,26])$ and thus most likely represent products of genes absent from the current assembly, whilst 103 (53.9\%) displayed nucleotide identities of between 97.099.5\% to genomic sequences. Given the presence within the majority of multi-membered subgroups of genomic sequences sharing $>97 \%$ identity (data not shown), it is not possible to conclude whether these 103 TRAV/ TRDV sequences represent allelic variants of genes identified in the genome or products of TRAV/TRDV genes absent from the current assembly. In contrast, the expressed TRAJ and TRDJ genes all corresponded to a genomic sequence - all 3 TRDJ genes and all functional TRAJ genes with the exception of TRAJ1, 6, 8-1, 41, 59, 60 were represented in the expressed TRA/TRD chains analysed.

\section{Discussion}

Gene duplication has been fundamental to the evolution of antigen-specific receptors, leading to the formation of the separate IG/TR loci $[7,27,28]$ and the expansion of the repertoire of $\mathrm{D}, \mathrm{J}$ and most notably $\mathrm{V}$ genes within these loci. Inter-loci and inter-species comparisons demonstrate that the extent of duplication that has occurred in different IG/TR loci during their evolution varies dramatically. The large number of bovine TRAV/TRDV genes identified in previous genomic analyses $[14,15]$ 


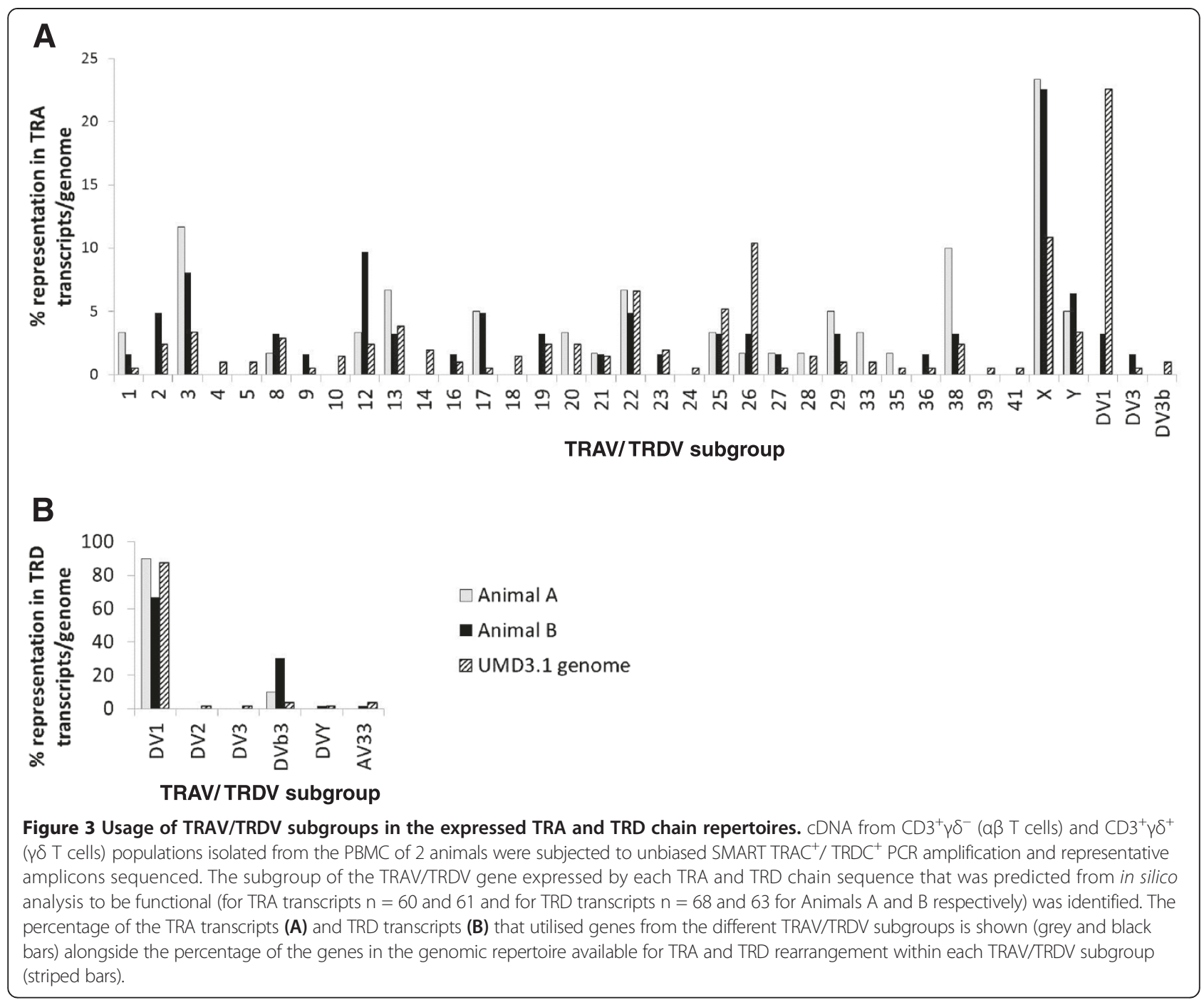

demonstrates that duplication within the bovine TRA/ TRD locus has been prolific. In this study we have used genomic and cDNA analyses to investigate the evolutionary basis for this dramatic expansion and its relationship with the expressed TRA and TRD chain repertoires.

The availability of an improved bovine genome assembly was critical to the study. The UMD3.1 assembly is more accurate than the Btau versions with a greater degree of completion, more contiguous sequence, corrections of SNPs and removal of erroneous segmental duplications [16]. Thus, although the catalogue of genes defined in our annotation is in general agreement with previous analyses $[14,15]$ there are substantive differences. Most significant of these are the identification of genes belonging to 5 novel bovine TRAV/TRDV subgroups (TRAV6, 34 and 37 and TRDV2 and Y) and a reduction in the total number of TRAV/TRDV genes identified (371 vs. 402 in [15]). Comparative analyses indicated that Btau_4.0 included many more TRAV/
TRDV genes showing 100\% nucleotide identity, suggesting the discrepancy in TRAV/TRDV gene number is largely attributable to the annotation of erroneous duplications in Btau_4.0. However, more pertinent to our study was the improved mapping of genes to the TRA/ TRD locus on chromosome 10 and the larger fragments of contiguous TRA/TRD locus sequence. Despite these improvements, the UMD3.1 assembly, like the previous Btau_4.0 and _3.0 assemblies, is i) derived from DNA taken from a father-daughter pairing, which due to the highly polymorphic nature of TRAV/TRDV genes, means that the potential for some proportion of the TRAV/TRDV expansion to be artefactual cannot be excluded, and ii) highly fragmented, with cDNA analysis suggesting that a substantial number of TRAV/TRDV genes are still missing. Consequently, definition of the total bovine TRAV/TRDV repertoire will be dependent on further work to obtain a completed assembly of the bovine TRA/TRD locus. 
The high level of similarity in the organisation and gene content of the $3^{\prime}$ end of the bovine, murine and human TRA/TRD loci, which contains the TRAJ, TRDJ, TRAC, TRDC genes and the main regulatory elements, indicates that since the divergence of the primate, rodent and artiodactyl lineages 100 million years ago (MYA) [29] there has been strong conservative evolution of this region. In direct contrast, the huge disparity in the numbers of TRAV/TRDV genes located in the $5^{\prime}$ end of the human, murine and bovine TRA/TRD loci describes a divergent evolutionary development. Expansion of the human TRAV/TRDV repertoire (54 genes) has been limited with only 7 of the 44 TRAV/TRDV subgroups having multiple-members and only TRAV8 (7 genes) having $>3$ members. In mice a higher proportion of subgroups are multi-membered (14 out of 28) and these are in general larger (TRAV6/TRDV9 is the largest subgroup, comprising 12 members) but the repertoire of 104 TRAV/TRDV genes is still modest in comparison to the 371 genes in cattle. Duplication appears to be a general feature of bovine TRAV/TRDV subgroups, with the majority (34 out of the 42) being multi-membered; however, the more remarkable feature is the extent of duplication within some of the subgroups - TRDV1 has 60 members, and TRAV22, 23, 25, 26 and $X$ all have $>20$ members. In this respect there are clear parallels with the bovine TRB locus where amplification of the TRBV repertoire is characterised by the prodigious expansion of certain subgroups (TRBV6 and 9) [8].

The location of $77.6 \%$ of bovine TRAV/TRDV genes in homology units demonstrates that homology unit replication has been the principal mechanism for expansion of the repertoire. In IG/TR loci expansion of the V gene repertoire by duplication of homology units incorporating genes from multiple subgroups is a common feature [7-9,30,31], although the extent to which this has occurred and its complexity differs markedly between loci. The identification in the UMD3.1 assembly of 10 putative homology units, ranging in size from 15 to $157 \mathrm{~Kb}$ and containing 2-17 TRAV/TRDV genes, many of which had undergone repeated replication, shows that the expansion of the bovine TRAV/TRDV repertoire has involved a particularly complex series of duplication events. In direct contrast, the majority of the murine TRAV/TRDV expansion is accounted for by a recent single duplication event in which a $\sim 410 \mathrm{~Kb}$ segment containing $40 \mathrm{TRAV} / \mathrm{TRDV}$ genes was replicated, whilst in the human TRA/TRD locus homology unit duplication has not been documented. Similarly, homology unit replication in other mammalian TR loci is much simpler than that described here. Due to the fragmented nature of the TRA/TRD locus in the UMD3.1 assembly, many of the homology unit copies analysed were incomplete and, with the exception of homology unit 2 (TRAV2-TRAV3), it was not possible to define the full extent of the homology units. Therefore, although our data clearly demonstrate the key role homology unit duplication has had in evolution of the bovine TRAV/TRDV repertoire, analysis of improved, more contiguous assemblies of the bovine TRA/TRD locus will be required to fully appreciate the extent and complexity of homology unit duplication.

In addition to a prolific 'birth' rate, bovine TRAV/TRDV genes also exhibit a high 'death' rate (37.2\% pseudogenes) compared to humans (19\%) and mice (16.5\%). There was marked variation in the percentages of non-functional genes between the multi-membered subgroups; for example amongst the 6 largest subgroups it ranged from 83.3\% (TRAV23) to 20\% (TRDV1). Although observed in IG V subgroups [32], the high proportion of pseudogenes seen in several of the bovine TRAV/TRDV subgroups, is rare in TR V subgroups. Based on the assumption that within the context of 'birth-and-death' evolution, homology unit replication is utilised to expand the functional TRAV/TRDV gene repertoire, the frequent co-localisation within homology units of genes from low (e.g. TRDV1 or TRAVX) and high (TRAV23 or TRAV18) 'death' rate subgroups, suggests that expansion of some high 'death' rate TRAV/TRDV subgroups may have occurred as a 'collateral' consequence of selection for juxtaposed low 'death' rate subgroups. Such 'collateral' expansion of V gene repertoires appears to be a feature of the evolution of other bovine IG/TR loci. Genomic analysis of the bovine IGLH locus suggests that the non-functional IGHV2 subgroup may have been expanded due to colocalisation of IGHV2 and functional IGHV1 genes in a duplicated homology unit [33] and similarly in the IGL locus many members of the expanded but predominantly non-functional IGLV5 subgroup are found juxtaposed to functional IGLV1 subgroup 1 genes [34]. Many bovine TRAV/TRDV pseudogenes in equivalent/analogous locations within different replicates of a homology unit share a common mutational lesion (e.g. TRAV8 genes in replicates of homology unit 9 all have an identical 4 bp deletion in the $\mathrm{V}$ exon) whilst conversely there are examples of analogous genes in homology unit replicates being a mixture of pseudogenes with non-identical lesions and functional genes (e.g. TRAV24 genes in homology unit 9). This indicates that 'collaterally' expanded genes may either be pseudogenes prior to duplication or may, due to absence of selection, lose functionality following replication. Notably, TRDV1 genes are co-located in 4 different homology units with different permutations of genes from 4 of the other massively expanded subgroups - TRAV22, 23, 25 and 26. Whilst the percentage of TRDV1 pseudogenes (20\%) is much lower than the bovine TRAV/TRDV average (37.2\%) that of the other subgroups is higher, ranging from 43.4-83.5\%. Thus, under the model of 'collateral' expansion, replication of 
homology units 7-10 and the consequent expansion of subgroups TRAV22, 23, 25 and 26 may have largely been due to evolutionary selection for a large functional TRDV1 repertoire. As these 5 subgroups together account for nearly half of the annotated bovine TRAV/TRDV genes, TRDV1 selection may have been a critical force in the evolution of the bovine TRAV/TRDV repertoire.

A unique feature of the TRAV/TRDV locus is the co-localisation and sharing of $\mathrm{V}$ genes available for rearrangement of 2 distinct antigen-specific receptor chains expressed by 2 distinct lineages of lymphocytes. Consequently, to understand the evolution of the bovine TRAV/TRDV gene repertoire in the context of the TR expressed by $\alpha \beta$ and $\gamma \delta$ T cells we undertook an analysis of the TRA and TRD transcripts expressed by these T cell subsets. Phylogenically defined TRAV/TRDV gene orthologues don't necessarily have the same capacity to partake in TRA and TRD rearrangements - for example mTRDV3 and hTRAV40 are phylogenetic orthologues but rearrange exclusively with TRDC and TRAC respectively. It was therefore necessary to first empirically evaluate TRAV/ TRDV subgroup usage in rearranged TRA and TRD transcripts using TRAV/TRDV subgroup-specific and TRAC/ TRDC specific primers. This analysis confirmed that each of the subgroups assigned as TRAV based on orthology to human TRAV subgroups were represented in rearranged TRA chain transcripts (with the exception of TRAV39) and that genes from all of the subgroups assigned as TRDV were demonstrated to be represented in TRD chain transcripts. Only genes from subgroups TRAV33, TRDV1, TRDV3 and TRDVb3 were expressed in both rearranged TRA and TRD chains, extending the previous observations of dual usage of these subgroups [17]. Thus, in contrast to humans and mice, dual usage of TRAV/TRDV genes in cattle makes a minimal contribution to diversifying the available TRDV germline repertoire but instead has been employed to further diversify the germline repertoire of $\mathrm{V}$ genes available for TRA rearrangement.

Commensurate with the number of TRAV/TRDV subgroups available for rearrangement, 'unbiased' transcript analysis demonstrated that the TRA repertoire uses genes from a diverse range of TRAV/TRDV subgroups, most of which are represented at $<5 \%$, whereas the TRD repertoire predominantly expressed TRDV1 genes, with minor contributions from a limited number of additional TRAV/TRDV subgroups. The dominance of TRDV1 in the expressed TRD repertoire suggests a functional advantage conferred by TRD chains utilising TRDV1 genes may have selected for the expansion of the TRDV1 genomic repertoire and consequently been a major determinant in the replication of homology units 7-10. Conversely the under-representation of TRAV26 (and TRDV1) genes in the expressed TRA repertoire suggests functional selection through TRA chain expression has not been a primary factor in replication of these homology units, but rather supports the concept that some TRAV subgroups have been expanded 'collaterally' as a consequence of being within the same homology units as TRDV1. However, over-representation of TRAV3 and TRAVX gene usage in the expressed TRA repertoire indicates that TRA selection has driven replication of other homology units (2, 5 and 6). As such the transcript data indicate that TRA and TRD selection may have driven expansion of different components of the genomic bovine TRAV/TRDV gene repertoire, with TRD selection playing an apparently disproportionately large role. The significance of TRD selection in shaping evolutionary development of the functional bovine TRAV/TRDV genomic repertoire is suggested by the marked increase in the proportion of Group 4 genes (preferential used in TRD chains) and concomitant decrease in the proportion of Group 1 genes (preferentially used by TRA chains) seen in cattle compared to mice and humans.

Although our studies have provided an insight into the potential mechanisms of the evolution of the bovine TRA/TRD locus, the biological factors that have led to the massive expansion of the TRAV/TRDV repertoire remain a matter of conjecture. Cattle, along with other artiodactyl species have proportionally higher numbers of circulating $\gamma \delta \mathrm{T}$ cells than humans and mice; this is assumed to reflect a greater immunological role for $\gamma \delta$ $\mathrm{T}$ cells in these species [35]. A parallel but apparently evolutionarily independent expansion of the TRDV1 subgroup in pigs (Sus scrofa) may signify that in artiodactyls common selective forces have led to high frequencies of $\gamma \delta \mathrm{T}$ cells and TRDV1 gene duplication. Incomplete knowledge on the functional roles of $\gamma \delta$ T cells and the ligands recognised by $\gamma \delta$ TR limit our ability to construct a hypothesis of the evolutionary advantage conferred to artiodactyls by the expansion of TRDV1 genes. However, it is well recognised in mice and humans that subsets of $\gamma \delta$ T cells defined by specific TRDV (and TRGV) gene expression have distinct functional phenotypes (reviewed in [36]). In addition to roles such as cytotoxicity and IFNy production, human $\mathrm{TRDV}^{+} \gamma \delta \mathrm{T}$ cells have been shown to express an immune-regulatory phenotype following stimulation with a TR-independent agonist [37]. Recent evidence demonstrating that $\gamma \delta$ T cells rather than FoxP3 ${ }^{+} \mathrm{CD} 4{ }^{+} \mathrm{T}$ cells contain the dominant regulatory $\mathrm{T}$ cell subset in cattle [38] raises the intriguing possibility that the requirement for a substantial immune-regulatory $\mathrm{T}$ cell population may have been a factor leading to the expansion of TRDV1 ${ }^{+} \gamma \delta$ T cells in cattle and other artiodactyls.

\section{Conclusion}

A comprehensive understanding of the evolutionary development of complex multi-gene families, such as the $\mathrm{V}$ genes of IG/TR loci requires integration of a variety of 
data. Previous studies cataloguing the bovine TRAV/ TRDV genes have demonstrated the unprecedented size of this repertoire but have not addressed the underlying evolutionary processes leading to its expansion. By combining data on gene characterisation, function, organisation and expression we have shown that the expansion of bovine TRAV/TRDV genes has been achieved primarily through a highly complex series of homology unit replications. We provide evidence indicating that as a consequence of this mode of replication a substantial proportion of the expanded TRAV gene repertoire may have occurred 'collaterally' as a consequence of positive selection for duplication of adjacent TRAV/TRDV segments, most notably TRDV1 genes. As TRD selection appears to be the main driver of TRDV1 expansion, this suggests that in bovine (and perhaps other artiodactyls) TRD selection may have been a dominant factor in the genomic expansion of a number of TRAV subgroups. Such data provide an important novel perspective of the evolutionary dynamics that have led to the development of the dramatically expanded bovine TRAV/TRDV repertoire and may have relevance to other IG/TR loci with enlarged V gene repertoires.

\section{Methods}

\section{Genome and sequence analysis}

The UMD3.1 genome assembly sequence was accessed through the ensembl website (http://www.ensembl.org). To identify bovine TRA/TRD gene segments, a series of BLASTn searches using the sequences of all human and murine TRAV/TRDV, TRDD, TRDJ, TRAJ, TRAC and TRDC genes (downloaded from the IMGT website (http://www.imgt.org)) and previously published bovine TRDV and TRDD sequences [17,21] were completed. Coordinates given in the gene annotation (Additional file 1) include the $\mathrm{L}$ exon, intron, $\mathrm{V}$ exon and $\mathrm{RS}$ of TRAV/TRDV genes, the splice site, RS and coding regions of the TRAJ, TRDJ and TRDD genes and the 4 exons of the TRAC and TRDC genes (including the polyA signal sequence). Basic sequence analysis, such as CLUSTALw alignments [39] and translations were conducted using the DNAsis Max v2.7 programme (MiraiBio, Almeda, CA, USA). In silico analysis of gene function considered the following parameters: i) presence of splice sites appropriate for RNA editing, ii) open reading frames which included codons for the conserved cysteine, tryptophan and cysteine residues at positions 23, 41 and 104 of TRAV/TRDV genes (IMGT unique numbering system [40]) and the canonical FGxG motif in TRAJ and TRDJ genes, iii) presence of a 23- and/or 12-RS sequence compatible with effective recombination $[41,42]$, and iv) location either on Chromosome 10 or an unassigned contig/ scaffold. Nucleotide identity analysis was performed using the Pipmaker and Multi-pipmaker programmes [43]. EST sequence data was derived from megablast searches of the NCBI Bos taurus EST trace archive (http://blast.ncbi.nlm. nih.gov) conducted with the nucleotide sequences of exon 1 of the bovine TRAC and TRDC genes in March 2013.

\section{Phylogenetic analyses}

Phylogenetic analysis was performed on the nucleotide sequence of the V-REGION (IMGT numbering 1-108) of TRAV/TRDV genes and the coding sequence of TRAJ and TRDJ genes of humans, mice and bovine (as identified in UMD3.1). Neighbour-joining method, minimum evolution and UPMGA analyses were performed with the MEGA5 software package [44] using the uncorrected nucleotide differences (p-distance), which provides better results when examining a large number of sequences which contain a relatively small number of nucleotides [45].

\section{CDNA analysis of rearranged TRA and TRD transcripts}

Blood from Holstein-Friesian animals was collected by jugular venu-puncture into EDTA and PBMC isolated by density gradient centrifugation over Ficoll-paque Plus (GE Healthcare, Little Chlafont, Bucks., UK) according to the manufacturer's instructions. $\alpha \beta$ and $\gamma \delta$ T cell populations were obtained by co-staining PBMC with monoclonal antibodies against bovine $\gamma \delta$ TR (GB21A - IgG2b isotype) and CD3 (MM1A - IgG1 isotype), both from VWRD, Pullman, WA, USA and then appropriate conjugated isotype-specific secondary antibodies (Molecular Probes, Invitrogen, Paisley, UK) prior to sorting into $\mathrm{CD}^{+} \gamma \delta^{+}$ ( $\gamma \delta \mathrm{T}$ cell) and $\mathrm{CD}^{+} \gamma \delta^{-}(\alpha \beta \mathrm{T}$ cell) populations on a FACSaria cell sorter (BD Biosciences, Oxford, UK). All animals used in this study were part of the Langhill Herd at the University of Edinburgh Farm. The work was approved by The Roslin Institute Animal Welfare and Ethical Review Body and conducted under license and in accordance with the UK government Animal (Scientific Procedures) Act 1986, UK.

For the TRAV/TRDV subgroup-specific PCR reactions total RNA was extracted using Tri-reagent (SigmaAldrich, Poole, Dorset, UK) and cDNA synthesised using the Reverse Transcription System (Promega, Madison, WI, USA) with priming by the Oligo $(\mathrm{dT})_{15}$ primer; both kits were used according to the manufacturer's instructions. Panels of PCRs were then conducted with each reaction using a single TRAV/TRDV subgroupspecific $5^{\prime}$ primer (Table 5) in combination with either a TRAC-(TRAC1 -GGGCTTCTCAGCTGGTACAC) or TRDC-(TRDC1 - CCCAGGTGAGATGGCAATAG) specific 3 ' primer. Individual reactions were composed of $1 \mu \mathrm{l}$ cDNA template, 10 pmol each of the relevant 5' and 3' primers, 0.5 units of Biotaq (Bioline, London, UK) and $2 \mu \mathrm{l}$ SM-0005 10× buffer (ABgene, Epsom, Surrey, UK) per $20 \mu \mathrm{l}$ reaction. Cycling conditions were $5 \mathrm{~min}$. at $95^{\circ} \mathrm{C}$, 
Table 5 Sequence of TRAV/TRDV subgroup-specific primers

\begin{tabular}{|c|c|}
\hline TRAV/TRDV subgroup & Subgroup-specific 5' primer sequence \\
\hline AV1 & CAGGAAAAGGCGTTAAGCAG \\
\hline AV2 & GGTCTCTTTGGAGGGAGCTG \\
\hline AV3 & CAGCCAGAAGCTGAGGTC \\
\hline AV4 & AGTGACCGTGCTCCTGAC \\
\hline AV8.1 & TGATGCTTGAGATGCTCCTG \\
\hline AV8.2 & CGGTCACATCAACGTCTCTG \\
\hline AV8.3 & ATTCCAGAGGCCAGTCAGTG \\
\hline AV9 & СTTCTCCAGGCTTAGTGACTG \\
\hline AV10 & TGAGTGGCAAAAACCAAGTG \\
\hline AV12 & CACAGTGGAGCAGAGTCCTG \\
\hline AV5 and 13 & CTTGTGGCT(A/G)CAGCTGGAC \\
\hline AV14 & AGGTGGTCGTGGCTTCACT \\
\hline AV16 & CAAGAGCCCAGACAGTGACTC \\
\hline AV17 & GAGAAGCTTCTGGCCCTG \\
\hline AV18 & GTTGTTACCCT(C/T)CCCGAGAAG \\
\hline AV19 & G(G/C)ATGTA(A/G)CCTTGAACTGTGC \\
\hline AV20 & CCAGGAGGGGGACAGTCT \\
\hline AV21 & GCCTGCTCATCCTITGGTTA \\
\hline AV22 & CTT(C/T)TGTTTGCCCAGGTTTG \\
\hline AV23 & AAGTGACCAA(G/C)AGCAGGTGA \\
\hline AV24 & CCCTTGCTGTGGGTTCAG \\
\hline AV25 & G(G/C)ACCAGTGTTGATCTTA(C/T)GGA \\
\hline AV26 & TCCATGGATT(A/G)T(G/C)CTGAAGG \\
\hline AV27 & TGGTCTITTGGATTCAACTGG \\
\hline AV28 & ACAAAGAAGAGTCTTGCTGAGTC \\
\hline AV29 & GCTTCAGTCTGACTGGGTG \\
\hline AV33 & GGCTGACAAAGTTACTGAAGC \\
\hline AV35 & GACATGTGTGAGTGCCCAAC \\
\hline AV36 & CCCCATCTCTGATTGTCCAT \\
\hline AV38 & CACAGTGACCCTGGACTGTA \\
\hline AV41 & AGGAAGGAGACCTCGTCACA \\
\hline AVX & GGCTCTC(A/C)TGACCCTGAACT \\
\hline AVY & GGTGGAGCAGA(A/G)TCCTTCAG \\
\hline DV1.1 & $(\mathrm{G} / \mathrm{C} / \mathrm{T}) \mathrm{C}(\mathrm{A} / \mathrm{T}) \mathrm{GCTCTGGGTG}(\mathrm{C} / \mathrm{T}) \mathrm{TCCT}$ \\
\hline DV1.2 & GTGGCCCAGA(A/G)(A/C/T)GTTACTCA \\
\hline DV2 & TCATCCACCTCACCCTCTTC \\
\hline DVY & CGTAACTGGAGGGAACTGGA \\
\hline DV3 & ATGTTCTCCCTGTGGGCTTC \\
\hline DVb3 & TCTGCCTTGGTTCCAACAAT \\
\hline
\end{tabular}

All of these primers were designed as part of this study based on sequences of functional members of the TRAV/TRDV subgroups identified in the UMD3.1 genome assembly, with the exception of DV3 which was published previously [17].
35 cycles of $\left(1 \mathrm{~min}\right.$. at $95^{\circ} \mathrm{C}, 1 \mathrm{~min}$. at $60^{\circ} \mathrm{C}, 1 \mathrm{~min}$. at $72^{\circ} \mathrm{C}$ ), and a final extension period of $5 \mathrm{~min}$. at $72^{\circ} \mathrm{C}$.

For the SMART PCR mRNA was extracted using the Dynabeads mRNA Direct Kit (Life Technologies, Paisley, UK) and cDNA synthesised with the SMARTer RACE cDNA Kit (Clontech, Paris, France) using TRAC- (TRAC2 TGTATTGGCATCCAGCATCG) and TRDC- (TRDC2 CGAGGTTTGTCCCATTTTTC) specific primers. PCR using the Phusion High-Fidelity PCR kit (New England BioLabs, Hitchin, UK) was then completed with the TRAC1/TRDC1 and the Long and Short UPM primers. Reactions were composed of Phusion HF amplification buffer, 3\% DMSO, $0.2 \mathrm{mM}$ dNTPs, $0.1 \mu \mathrm{M}$ Long UPM, $0.5 \mu \mathrm{M}$ Short UPM, $0.5 \mu \mathrm{M}$ of internal TRAC or TRDC primer, Phusion Hot Start DNA polymerase $(2 \mathrm{U} / 100 \mu \mathrm{l}$ final reaction volume) and cDNA $(1 \mu \mathrm{l} / 100 \mathrm{ul}$ final reaction volume). Cycling conditions were $30 \mathrm{~s}$ at $98^{\circ} \mathrm{C}, 30-$ cycles of $\left(98^{\circ} \mathrm{C}\right.$ for $10 \mathrm{~s}, 57^{\circ} \mathrm{C}$ for $20 \mathrm{~s}, 72^{\circ} \mathrm{C}$ for $\left.20 \mathrm{~s}\right)$, and a final extension period of $5 \mathrm{~min}$. at $72^{\circ} \mathrm{C}$.

PCR products were visualised in 1.5\% TAE-agarose gels, purified using Qiaquick gel extraction kit (Qiagen, Crawley, UK) and cloned into pGEM-T Easy (Promega, Madison, WI, USA). Selected cloned products were sequenced (Genepool, University of Edinburgh, UK) to permit verification of the sequence of the amplified TRA/TRD chains. One hundred and twenty-six partial TRA and TRD transcripts, including representatives from each of the expressed functional TRAV/TRDV subgroups have been submitted to Genbank (accession numbers JX065635-JX065739 and JX101710-101720).

\section{Availability of supporting data}

The nucleotide and phylogenetic datasets supporting the results of this article are available in GenBank (http:// www.ncbi.nlm.nih.gov/ - accession numbers JX065635JX065739 and JX101710-101720) and Dryad (http://doi. org/10.5061/dryad.h8j92) respectively. The primary sequence data, as derived from UMD3.1 assembly of the bovine genome and accessed through ensembl (http:// www.ensembl.org/index.html) is in Additional file 5.

\section{Nomenclature}

Without a fully assembled TRA/TRD locus it was not possible to implement the approved nomenclature system which requires knowledge of the genomic order of genes from the $5^{\prime}$ to the $3^{\prime}$ end of the locus [46]. Bovine TRAV/TRDV subgroups have been named according to the orthologous human subgroups where appropriate and an alphabetic designation where this was not possible. Members in the $\mathrm{V}$ gene subgroups have been given alphabetic rather than numeric designations, similar to systems employed in previous work [8,14], although there is no correspondence between the TRDV1 nomenclature employed here and in [14]. The TRDD and TRDJ 
nomenclature is consistent with previously published work and TRAJ genes have been assigned numbers according to their $5^{\prime}$ to $3^{\prime}$ order in the genome.

\section{Additional files}

\section{Additional file 1: Location of TRA/TRD genes and regulatory} elements in UMD3.1. For each gene the name, type of gene segment, gene orientation (ori.), chromosomal location, start and stop co-ordinates, chromosome orientation (chrom. ori.), contig number and predicted functional competency are shown. Sequences matching TRA/TRD genes marked by an asterisk (as a suffix to the gene name) have been described in previous annotations - Reinink and Van Rhijn [15] and Herzig et al. [14], or in the IMGT database (July 2014 - based on data from Herzig et al. [14]). The coloured blocks represent the locations of homology units (see Additional file 8)

Additional file 2: Neighbour-joining phylogenetic tree of all murine, human and bovine (from the UMD3.1 assembly) TRAV/ TRDV genes. Analysis of the nucleotide sequence of the V-REGION (IMGT nomenclature) following pairwise deletion to remove gaps in the alignment. The final dataset included 400 positions. The sequence of bTRBV3a was used to root the tree. Based on a 1000 boot strap replicates the phylogenetically inferred orthologous TRAV/TRDV subgroups were supported by percentage bootstrap values $\left(P_{B}\right)$ of $>90 \%$ in all cases except for bTRAV38 $\left(P_{B}=83 \%\right)$, bTRAV5 $\left(P_{B}=59 \%\right)$, bTRAV13 $\left(P_{B}=85 \%\right)$ and bTRAV8 $\left(P_{B}=68 \%\right)$. Sequence identity between bovine and human genes in orthologous groups ranged from $63.1-84.9 \%$, sufficient to assign them as inter-species orthologues [47]. Generally the phylogenetically defined bovine TRAV/TRDV subgroups adhered to the convention of members sharing $>75 \%$ nucleotide identity $[25,26]$. However, within both the bTRDV1 and bTRAV 8 subgroups identity between some members was $<75 \%$ (down to 68.0 and $69.7 \%$ respectively) and conversely the identity between some bTRAV5/13 and some bTRAVX/18 members was $>75 \%$. Due to difficulties in alignment the following genes were excluded from the analysis i) bovine genes for which only incomplete or partial genomic sequences were available, ii) bTRAV11a - due to the presence of a large insert and iii) mTRAV15-3, mTRAV15D-3, hTRAV8.5. h= human, $\mathrm{b}=$ bovine and $\mathrm{m}=$ murine.

Additional file 3: Comparison of the annotated TRA/TRD genes with previously published annotations of the bovine TRA/TRD locus and data available in the IMGT website (July 2014). Summary of comparisons with the TRA/TRD gene repertoires identified by Reinink and Van Rhijn [15], Herzig et al. [14] and present in the IMGT database (July 2014). Also summarised are the number of TRA/TRD genes which have been identified in previous annotation studies.

Additional file 4: Annotation of the exons and RS sequences of the TRA/TRD genes. The coordinates of the (A) L-exons, V-exons and RS of each TRAV/TRDV gene, (B and C) RS and J-gene of each TRAJ and TRDJ gene, (D) RS and D-genes of each TRDD gene and (E) exons of the TRAC and TRDC gene are detailed.

Additional file 5: Sequences of (A) Chr10: 22100001-25700000, (B) Chr10:60200001-60300000, (C) Chr9:71300001-71400000 and (D) Contig DAAA0206600. This provides the primary sequence resources from which the sequences of the TRA/TRD genes annotated can be extracted.

Additional file 6: Neighbour-joining phylogenetic tree of all murine, human and bovine (from the UMD3.1 assembly) TRAJ genes. Analysis of the nucleotide sequence of the coding domain of TRAJ genes following pairwise deletion to remove gaps in the alignment. The final dataset had a total of 85 positions. Based on a 1000 boot strap replicates the orthologous TRAJ genes from mouse, human and cattle (where all genes were functional) formed phylogenetic groups supported by percentage bootstrap values $\left(P_{B}\right)$ of $>75 \%$ (with the exception of TRAJ6 $\left(P_{B}=54 \%\right), 9(53 \%)$ and $\left.48(67 \%)\right)$. $P_{B}$ values supporting the phylogenetic groups of TRAJ orthologues which included non-functional members were generally high but in several cases were $<50 \%$. $h=$ human, $b=$ bovine and $m=$ murine. The formation of 'triads' composed of single genes from each species (except TRAJ10 which lacks a murine gene and TRAJ8 which contains 2 bovine genes) that have the same relative position in the genome (as denoted by their numerical designation) demonstrates conserved synteny. The level of nucleotide identity between orthologous bovine and human TRAJ genes ranges from $63.2 \%$ to $95.2 \%$.

Additional file 7: Sequence alignment of regulatory elements in the 3 ' end of the bovine (from the UMD3.1 assembly), murine and human TRA/TRD locus. Sequences of defined transcription factor binding sites are shown in grey highlight. Nucleotide identity between orthologous sequences is shown by dashes and gaps by dots. (A) Alignment of the human, murine and putative bovine Ea sequences. Protein binding regions in the core human Ea (Ta1-4) are indicated by arrows. The 300 bp sequence of the putative bovine Ea shares $87.5 \%$ and $78.2 \%$ nucleotide identity with the human and murine Ea. Within the core Ta1-Ta2 fragment of the Ea, which constitutes the DNA scaffold for the generation of the nucleo-protein structure termed the 'enhanceosome' that is critical for Ea function [48-50], the CREB, TCF-1/LEF-1 and Ets binding sites show absolute conservation between the bovine, human and murine sequences. Simultaneous occupancy of these sites is a minimal requirement for Ea activity. Numerous other transcription factor binding sites which have been shown to be occupied in the Ea and play a role in appropriate regulation of Ea function [51-53] are also conserved in the putative bovine Ea sequence. (B) Alignment of the human, murine and putative bovine $E \delta$ sequences. Protein binding regions in the core human $\mathrm{E} \delta(\mathrm{S} \varepsilon 3$ and $\mathrm{S} \varepsilon 4$ ) are indicated by arrows. The 70 bp sequence spanning the essential $S \varepsilon 3$ and S\&4 core of the enhancer [54] shows $77.1 \%$ and $72.8 \%$ nucleotide identity to the corresponding human and murine sequences. The CBF/PEPB2 and c-myb binding sites within $S \varepsilon 3$ which are critical for the formation and function of the $\mathrm{E} \delta$ 'enhanceosome' [55] are conserved in the bovine sequence as are 2 GATA-3 binding sequence in $S \varepsilon 4$.

Additional file 8: A -TRAV/TRDV homology units identified within UMD3.1. Details of the replicons of the 10 putative homology units are given. Each homology unit has been ascribed a colour code that can be used to examine the position of homology units within Additional file 1. Each copy of the homology unit has been designated a number (e.g. Homology Unit 1.1) to enable correspondence with the nucleotide identity analysis presented in Additional file 9. For each homology unit the TRAV/TRDV subgroup motif is shown (TRAV subgroups unless otherwise specified) and for each homology unit copy the specific genes and their functionality ( $F$ - functional, PS - pseudogene, I - incomplete) are detailed. Where present on the same contig, genes flanking the putative homology unit replicates are shown on a green background. However, if homology unit copies appear to extend beyond the limits of the contig on which they're located this is shown as END on a red background. Three sequences show high identity with both homology unit 5 and 6 - these are designated as ambiguous (AMB in Additional file 1). Genes within homology unit 5 replicates shown on an orange background may represent a variant of the homology unit that has arisen during different replicative iterations. Dashes within homology unit replicant gene sequences represent absent genes - most likely as consequences of post-replication insertion/deletion. Non-contiguous sequences have generally not been used for this analysis, but in circumstances where joining non-contiguous sequences rationalised the number of fragments of homology units identified, exceptions have been made (genes flanking breaks in contiguous sequence are shown in red italicised script on a yellow background). Homology unit replicates identified by an asterisk were used as reference sequences for nucleotide identity analysis using the Pipmaker/ Multipipmaker programmes; boxed subgroups in TRAV/TRDV subgroup homology unit motifs are absent from the reference sequences.

Additional file 9: B - Nucleotide identity analysis of putative homology units. Nucleotide identity analysis was conducted using the Pipmaker and Multipipmaker programmes as described in Materials and Methods. For homology units with only 2 replicates (homology units 1, 3 and 10) dotplots are shown and for homology units with $>2$ replicates (homology units 4, 5, 6, 7, 8 and 9) a summary of the Multipip output is shown. For homology unit 2 a dotplot covering the entire region of alternating TRAV2 and TRAV3 genes against itself is shown as this best 
exemplifies the multiple tandem repeats that have occurred. In dotplots diagonal lines represent areas of nucleotide identity; in the Multipip output areas of high nucleotide identity are represented by red colouring.

Additional file 10: A Genomic sequence of the bovine TRAJ genes identified in UMD3.1. The table shows the (i) RS sequence, (ii) reading frame of the coding sequence, (iii) nucleotide and predicted amino acid sequences of the coding sequence and (iv) the sequence of the $3^{\prime}$ splice site. The canonical FGXG motif of the TRAJ sequence is shown with yellow highlighting. TRAJ genes that are predicted to be pseudogenes are shown with their name and the identified defect highlighted in red. B - Summary of non-functional TRAJ gene segments in UMD3.1. Summary of the lesions identified in TRAJ genes that are considered to render them non-functional.

Additional file 11: Summary of non-functional TRAV/TRDV gene segments in UMD3.1. The lesions identified in TRAV/TRDV genes which are considered to render them non-functional are summarised.

\section{Abbreviations}

Ig: Immunolobulin; IMGT: IMGT®, the international ImMunoGeneTics information system ${ }^{\otimes}$; RS: Recombination signal sequence; TR: T cell receptor; TRA: T cell receptor alpha; TRD: T cell receptor delta; V: Variable gene segment; J: Joining gene segment; D: Diversity gene segment; C: Constant gene segment.

\section{Competing interests}

The authors declare that they have no competing interests.

\section{Authors' contributions}

TC conceived the study and performed the genome analysis; TC, KD and CL performed the CDNA analysis. TC prepared the manuscript with contributions from WM. All authors read and approved the manuscript.

\section{Acknowledgements}

We would like to acknowledge funding from the following sources. TC, KD, $\mathrm{CL}$ and WM were funded through grant No. BB/H009515/1 from the Department for International Development (UK government) and Biotechnology and Biological Sciences Research Council UK awarded to Prof. W.I. Morrison.

\section{Author details}

${ }^{1}$ The Roslin Institute and Royal (Dick) School of Veterinary Studies, The University of Edinburgh, Easter Bush, Midlothian EH25 9RG, Scotland, UK. ${ }^{2}$ The Moredun Group, Pentlands Science Park, Bush Loan, Penicuik, Midlothian, Scotland EH26 OPZ, UK.

Received: 14 April 2014 Accepted: 4 November 2014

Published: 19 November 2014

\section{References}

1. McBlane JF, van Gent DC, Ramsden DA, Romeo C, Cuomo CA, Gellert M, Oettinger MA: Cleavage at a V(D)J recombination signal requires only RAG1 and RAG2 proteins and occurs in two steps. Cell 1995, 83(3):387-395

2. Davis MM, Bjorkman PJ: T-cell antigen receptor genes and T-cell recognition. Nature 1988, 334(6181):395-402.

3. Nikolich-Zugich J, Slifka MK, Messaoudi I: The many important facets of T-cell repertoire diversity. Nat Rev Immunol 2004, 4(2):123-132.

4. Rowen L, Koop BF, Hood L: The complete 685-kilobase DNA sequence of the human beta T cell receptor locus. Science 1996, 272(5269):1755-1762

5. Osipovich O, Oltz EM: Regulation of antigen receptor gene assembly by genetic-epigenetic crosstalk. Semin Immunol 2010, 22(6):313-322.

6. Schlissel MS: Regulating antigen-receptor gene assembly. Nat Rev Immunol 2003, 3(11):890-899.

7. Glusman G, Rowen L, Lee I, Boysen C, Roach JC, Smit AF, Wang K, Koop BF, Hood L: Comparative genomics of the human and mouse T cell receptor loci. Immunity 2001, 15(3):337-349.
8. Connelley T, Aerts J, Law A, Morrison WI: Genomic analysis reveals extensive gene duplication within the bovine TRB locus. BMC Genomics 2009, 10:192.

9. Mineccia M, Massari S, Linguiti G, Ceci L, Ciccarese S, Antonacci R: New insight into the genomic structure of dog T cell receptor beta (TRB) locus inferred from expression analysis. Dev Comp Immunol 2012, 37(2):279-293

10. Nei M, Gu X, Sitnikova T: Evolution by the birth-and-death process in multigene families of the vertebrate immune system. Proc Natl Acad SCi U S A 1997, 94(15):7799-7806.

11. Nei M, Rooney AP: Concerted and birth-and-death evolution of multigene families. Annu Rev Genet 2005, 39:121-152.

12. Su C, Nei M: Evolutionary dynamics of the T-cell receptor VB gene family as inferred from the human and mouse genomic sequences. Mol Biol Evol 2001, 18(4):503-513.

13. Lefranc M-P, Giudicelli V, Ginestoux C, Jabado-Michaloud J, Folch G, Bellahcene F, Wu Y, Gemrot E, Brochet X, Lane J, Regnier L, Ehrenmann F, Lefranc G, Duroux P: IMGT ${ }^{\oplus}$, the international ImMunoGeneTics information system ${ }^{\circledR}$. Nucl Acids Res 2009, 37:D1006-D1012.

14. Herzig CT, Lefranc MP, Baldwin CL: Annotation and classification of the bovine T cell receptor delta genes. BMC Genomics 2010, 11:100.

15. Reinink P, Van Rhijn I: The bovine $T$ cell receptor alpha/delta locus contains over $400 \mathrm{~V}$ genes and encodes $\mathrm{V}$ genes without CDR2. Immunogenetics 2009, 61(7):541-549.

16. Zimin AV, Delcher AL, Florea L, Kelley DR, Schatz MC, Puiu D, Hanrahan F, Pertea G, Van Tassell CP, Sonstegard TS, Marçais G, Roberts M, Subramanian $P$, Yorke JA: A whole-genome assembly of the domestic cow, Bos taurus. Genome Biol 2009, 10(4):R42.

17. Herzig CT, Blumerman SL, Baldwin CL: Identification of three new bovine T-cell receptor delta variable gene subgroups expressed by peripheral blood T cells. Immunogenetics 2006, 58(9):746-757.

18. Blumerman SL, Herzig CT, Rogers AN, Telfer JC, Baldwin CL: Differential TCR gene usage between WC1- and WC1+ ruminant gammadelta T cell subpopulations including those responding to bacterial antigen. Immunogenetics 2006, 58(8):680-692.

19. Hein WR, Dudler L: TCR gamma delta + cells are prominent in normal bovine skin and express a diverse repertoire of antigen receptors. Immunology 1997, 91(1):58-64.

20. Ishiguro N, Aida Y, Shinagawa T, Shinagawa M: Molecular structures of cattle T-cell receptor gamma and delta chains predominantly expressed on peripheral blood lymphocytes. Immunogenetics 1993, 38(6):437-443.

21. Van Rhijn I, Spiering R, Smits M, van Blokland MT, de Weger R, van Eden W, Rutten VP, Koets AP: Highly diverse TCR delta chain repertoire in bovine tissues due to the use of up to four $D$ segments per delta chain. Mol Immunol 2007, 44(12):3155-3161.

22. Ishiguro N, Tanaka A, Shinagawa M: Sequence analysis of bovine T-cell receptor alpha chain. Immunogenetics 1990, 31(1):57-60.

23. Lahmers KK, Norimine J, Abrahamsen MS, Palmer GH, Brown WC: The CD4+ $T$ cell immunodominant Anaplasma marginale major surface protein 2 stimulates gammadelta $T$ cell clones that express unique $T$ cell receptors. J Leukoc Biol 2005, 77(2):199-208.

24. Quigley MF, Almeida JR, Price DA, Douek DC: Unbiased molecular analysis of T cell receptor expression using template-switch anchored RT-PCR. Curr Protoc Immunol 2011, Chapter 10:Unit10-Unit33.

25. Arden B, Clark SP, Kabelitz D, Mak TW: Mouse T-cell receptor variable gene segment families. Immunogenetics 1995, 42(6):501-530.

26. Arden B, Clark SP, Kabelitz D, Mak TW: Human T-cell receptor variable gene segment families. Immunogenetics 1995, 42(6):455-500.

27. Litman GW, Rast JP, Fugmann SD: The origins of vertebrate adaptive immunity. Nat Rev Immunol 2010, 10(8):543-553.

28. Richards $\mathrm{MH}$, Nelson $\mathrm{J}$ : The evolution of vertebrate antigen receptors: $a$ phylogenetic approach. Mol Biol Evol 2000, 17(1):146-155.

29. Hedges SB, Dudley J, Kumar S: TimeTree: a public knowledge-base of divergence times among organisms. Bioinformatics 2006, 22(23):2971-2972.

30. Bosc N, Lefranc MP: The mouse (Mus musculus) T cell receptor alpha (TRA) and delta (TRD) variable genes. Dev Comp Immunol 2003, 27(6-7):465-497.

31. Sitnikova T, Su C: Coevolution of immunoglobulin heavy- and light-chain variable-region gene families. Mol Biol Evol 1998, 15(6):617-625. 
32. Lefranc MP: IMGT, the international ImMunoGeneTics database. Nucleic Acids Res 2001, 29(1):207-209.

33. Niku M, Liljavirta J, Durkin $K$, Schroderus E, livanainen A: The bovine genomic DNA sequence data reveal three IGHV subgroups, only one of which is functionally expressed. Dev Comp Immunol 2012, 37(3-4):457-461.

34. Ekman A, Niku M, Liljavirta J, livanainen A: Bos taurus genome sequence reveals the assortment of immunoglobulin and surrogate light chain genes in domestic cattle. BMC Immunol 2009, 10:22.

35. Antonacci R, Lanave C, Del Faro L, Vaccarelli G, Ciccarese S, Massari S: Artiodactyl emergence is accompanied by the birth of an extensive pool of diverse germline TRDV1 genes. Immunogenetics 2005, 57(3-4):254-266.

36. Pang DJ, Neves JF, Sumaria N, Pennington DJ: Understanding the complexity of gammadelta T-cell subsets in mouse and human. Immunology 2012, 136(3):283-290.

37. Kress E, Hedges JF, Jutila MA: Distinct gene expression in human Vdelta1 and Vdelta2 gammadelta T cells following non-TCR agonist stimulation. Mol Immunol 2006, 43(12):2002-2011.

38. Hoek A, Rutten VP, Kool J, Arkesteijn GJ, Bouwstra RJ, Van Rhijn I, Koets AP: Subpopulations of bovine WC1(+) gammadelta T cells rather than CD4 (+)CD25(high) Foxp3(+) T cells act as immune regulatory cells ex vivo. Vet Res 2009, 40(1):6.

39. Thompson JD, Higgins DG, Gibson TJ: CLUSTAL W: improving the sensitivity of progressive multiple sequence alignment through sequence weighting, position-specific gap penalties and weight matrix choice. Nucleic Acids Res 1994, 22(22):4673-4680

40. Lefranc MP, Pommie C, Ruiz M, Giudicelli V, Foulquier E, Truong L, Thouvenin-Contet $\mathrm{V}$, Lefranc G: IMGT unique numbering for immunoglobulin and T cell receptor variable domains and Ig superfamily V-like domains. Dev Comp Immunol 2003, 27(1):55-77.

41. Akamatsu Y, Tsurushita N, Nagawa F, Matsuoka M, Okazaki K, Imai M, Sakano H: Essential residues in V(D)J recombination signals. J Immunol 1994, 153(10):4520-4529.

42. Hesse JE, Lieber MR, Mizuuchi K, Gellert M: V(D)J recombination: a functional definition of the joining signals. Genes Dev 1989, 3(7):1053-1061.

43. Schwartz S, Zhang Z, Frazer KA, Smit A, Riemer C, Bouck J, Gibbs R, Hardison R, Miller W: PipMaker-a web server for aligning two genomic DNA sequences. Genome Res 2000, 10(4):577-586.

44. Tamura K, Peterson D, Peterson N, Stecher G, Nei M, Kumar S: MEGA5: molecular evolutionary genetics analysis using maximum likelihood, evolutionary distance, and maximum parsimony methods. Mol Biol Evol 2011, 28(10):2731-2739.

45. Nei M, Kumar S: Molecular evolution and phylogenetics. Oxford, UK: Oxford University Press; 2000.

46. Lefranc MP: Nomenclature of the human T cell receptor genes. Curr Protoc Immunol 2001, Appendix 1:Appendix 10.

47. Lai $E$, Concannon $P$, Hood L: Conserved organization of the human and murine T-cell receptor beta-gene families. Nature 1988, 331(6156):543-546

48. Giese K, Kingsley C, Kirshner JR, Grosschedl R: Assembly and function of a TCR alpha enhancer complex is dependent on LEF-1-induced DNA bending and multiple protein-protein interactions. Genes Dev 1995, 9(8):995-1008.

49. Hernandez-Munain C, Roberts JL, Krangel MS: Cooperation among multiple transcription factors is required for access to minimal T-cell receptor alpha-enhancer chromatin in vivo. Mol Cell Biol 1998, 18(6):3223-3233.

50. Roberts JL, Lauzurica P, Krangel MS: Developmental regulation of VDJ recombination by the core fragment of the $T$ cell receptor alpha enhancer. J Exp Med 1997, 185(1):131-140.

51. Balmelle N, Zamarreno N, Krangel MS, Hernandez-Munain C: Developmental activation of the TCR alpha enhancer requires functional collaboration among proteins bound inside and outside the core enhancer. J Immunol 2004, 173(8):5054-5063.

52. Hernandez-Munain C, Sleckman BP, Krangel MS: A developmental switch from TCR delta enhancer to TCR alpha enhancer function during thymocyte maturation. Immunity 1999, 10(6):723-733.

53. Spicuglia S, Payet D, Tripathi RK, Rameil P, Verthuy C, Imbert J, Ferrier $P$, Hempel WM: TCRalpha enhancer activation occurs via a conformational change of a pre-assembled nucleo-protein complex. EMBO J 2000, 19(9):2034-2045.
54. Redondo JM, Pfohl JL, Krangel MS: Identification of an essential site for transcriptional activation within the human T-cell receptor delta enhancer. Mol Cell Biol 1991, 11(11):5671-5680.

55. Hernandez-Munain C, Krangel MS: Distinct roles for c-Myb and core binding factor/polyoma enhancer-binding protein 2 in the assembly and function of a multiprotein complex on the TCR delta enhancer in vivo. J Immunol 2002, 169(8):4362-4369.

doi:10.1186/1471-2164-15-994

Cite this article as: Connelley et al: Genomic analysis offers insights into the evolution of the bovine TRA/TRD locus. BMC Genomics 2014 15:994.

\section{Submit your next manuscript to BioMed Central and take full advantage of:}

- Convenient online submission

- Thorough peer review

- No space constraints or color figure charges

- Immediate publication on acceptance

- Inclusion in PubMed, CAS, Scopus and Google Scholar

- Research which is freely available for redistribution

Submit your manuscript at www.biomedcentral.com/submit
C Biomed Central 